\title{
Information Variability Impacts in Auctions
}

\author{
Justin Jia • Ronald M. Harstad · \\ Michael H. Rothkopf
}

the date of receipt and acceptance should be inserted later

\begin{abstract}
A wide variety of auction models exhibit close relationships between the winner's expected profit and the expected difference between the highest and second-highest order statistics of bidders' information, and between expected revenue and the second-highest order statistic of bidders' expected asset values. We use stochastic orderings to see when greater environmental variability of bidders' information enhances expected profit and expected revenue.
\end{abstract}

Keywords auctions, revenue, expected profit, order statistics, stochastic orderings (JEL:D44)

In the usual formulation of a game of incomplete information, each player privately observes his type, with each type drawn from a distribution that is common knowledge. Auction models typically follow this specification, either with types drawn independently, or drawn from affiliated distributions; independence conditional on a stochastic common value is a frequent example. A bidder values the auctioned asset as some commonly known function of the profile of types, with common-value auctions and private-values auctions (in which each bidder's value is his type) as special cases.

Typically, the equilibrium examined in these models exhibits a positive expected profit conditional on winning. ${ }^{1}$ The winner's profit results from the presence of private information: his estimate of asset value, properly conditioned on his type and on the event of winning, is an estimate for which other

\footnotetext{
Justin Jia

Smeal College of Business, Pennsylvania State University. E-mail: zuj100@psu.edu

Ronald M. Harstad

Department of Economics, University of Missouri. E-mail: HarstadR@missouri.edu

Michael H. Rothkopf

Smeal College of Business, Pennsylvania State University, deceased February 2008.

1 The exceptions stem from various heroic assumptions yielding full surplus extraction, cf. Mares and Harstad (2007) and references therein.
} 
bidders have but an imperfect substitute. The most widely studied case is independently drawn types; then under mild assumptions, Myerson (1981) shows that equilibrium expected revenue for any standard auction form is equal to the second-highest expected value estimate, and expected profit conditional on winning equals the difference between the highest and second-highest expected value estimates. This paper examines the relationship between the variability of the type distributions and these expected estimates, illuminating comparisons across auction environments where bidders' private information is less dispersed in one, and dispersed more widely in the other. ${ }^{2}$

To normalize to zero the seller's value of the auctioned asset is usual, harmless, and assumed throughout. The gains from trade are then equal to the highest expected value estimate. Except in virtually pathological cases, more widely dispersed private information increases the gains from trade. We explore assumptions determining whether the seller, and whether the winning bidder, shares in these increased gains.

In some auction settings, variability of bidders' information may be partially under the bid-taker's control: a seller may decide to reveal greater or lesser information about technical specifications of an auctioned asset; a buyer seeking bids for construction of a skyscraper may choose to offer insurance against price increases for certain raw materials as part of the auction specifications.

Sections 1 and 2 examine independently-distributed information; affiliation is considered following. Most proofs are relegated to the appendix.

\section{The Winning Bidder's Expected Profit}

This analysis contrasts a benchmark environment where any of the $n$ bidders observes a type $X$ drawn i.i.d. from nondegenerate distribution function $F$ with a more-dispersed-information environment where any bidder observes a type $Z=X+Y$, with the added randomness $Y$ distributed (independently) as $G .{ }^{3}$ Let $\mathfrak{X}=\left(X_{(1)}, \ldots, X_{(n)}\right)$ denote the order statistics (in descending order) of a sample of size $n$ from the population random variable $X$, and $\mathfrak{Z}=\left(Z_{(1)}, \ldots, Z_{(n)}\right)$, correspondingly. The characterization sought, Dispersion Benefits Bidders $(D B B)$, is then formally:

$$
E\left[X_{(1)}-X_{(2)}\right] \leq E\left[Z_{(1)}-Z_{(2)}\right] .
$$

Several types of stochastic orderings play roles:

\footnotetext{
${ }^{2}$ For concreteness, the text develops the case where bidders compete to buy from a bidtaking seller; completely corresponding results about the variability of (1) the second-lowest order statistic, and (2) the difference between the lowest and second-lowest order statistics, illuminate expected project cost and expected profit in the case where bidders compete to sell to a bid-taking buyer.

3 The incremental dispersion need not be additive: $Z$ can simply be more dispersed than $X$, with $Y=X-Z$ by definition.
} 
1. $X$ is stochastically smaller than $Y\left(\operatorname{read}, X \leq{ }_{s t} Y\right)$ if $\bar{F}(t) \leq \bar{G}(t) \forall t .{ }^{4}$

2. $X$ is less dispersed than $Y\left(X \leq_{\text {disp }} Y\right)$ if $0<\alpha<\beta<1 \Longrightarrow F^{-1}(\beta)-$ $F^{-1}(\alpha) \leq G^{-1}(\beta)-G^{-1}(\alpha)$ (Shaked 1982).

3. $X$ is smaller than $Y$ in the excess wealth order $\left(X \leq_{e w} Y\right)$ if $p \in(0,1) \Longrightarrow$ $\int_{F^{-1}(p)}^{\infty} \bar{F}(x) d x \leq \int_{G^{-1}(p)}^{\infty} \bar{G}(x) d x$ (Shaked and Shanthikumar 2006).

4. $X$ is smaller than $Y$ in the increasing convex order $\left(X \leq_{i c x} Y\right)$ if $\int_{t}^{\infty} \bar{F}(x) d x \leq$ $\int_{t}^{\infty} \bar{G}(x) d x \forall t$ (Ross 1996).

Theorem 1 Either (1) X has a log-concave density ${ }^{5}$ or (2) $X$ has an increasing failure rate $(I F R)^{6}$ is sufficient for $D B B$.

Proof Preliminaries: [a] $X$ satisfies $X \leq_{\text {disp }} X+Y$ for $Y$ independent of $X$ if and only if $X$ has a log-concave density. [b] Let $V_{(i)}=X_{(i)}-X_{(i+1)}$ and $W_{(i)}=Z_{(i)}-Z_{(i+1)}, i=1,2, \ldots, n .^{7}$ If $X \leq_{\text {disp }} Z$, then $V_{(i)} \leq_{s t} W_{(i)}$, $i=1,2, \ldots, n$. [c] $X$ satisfies $X \leq_{e w} X+Y$ for $Y$ independent of $X$ if and only if $X$ is IFR. [d] If $X \leq_{e w} Z$ then $X_{(1)}-X_{(k)} \leq_{i c x} Z_{(1)}-Z_{(k)}$ for $k=2,3, \ldots, n{ }^{8}$

If $X$ has a log-concave density, then (by [a]) $X \leq_{\text {disp }} X+Y$, and (by [b]) $V_{(1)} \leq_{s t} W_{(1)}$. Now $E\left[V_{(1)}\right] \leq E\left[W_{(1)}\right]$ follows from $\left\{A \leq_{s t} B\right\} \Longrightarrow$ $\{E[A] \leq E[B]\}$. If $X$ is IFR, then (by [c]) $X \leq_{\text {ew }} X+Y$, and (by [d]) $X_{(1)}-X_{(2)} \leq_{i c x} Z_{(1)}-Z_{(2)}$, which is a stronger result than $E\left[X_{(1)}-X_{(2)}\right] \leq$ $E\left[Z_{(1)}-Z_{(2)}\right] \cdot{ }^{9}$

Many commonly used distribution families have log-concave densities. These include uniform, normal, logistic, extreme value, exponential, Laplace, chisquared $(c \geq 2)$, Weibull $(c \geq 1)$, power function $(\beta \geq 1)$, gamma $(m \geq 1)$ and beta $(a \geq 1, b \geq 1)$ (See Bagnoli and Bergstrom 2005 for a more extensive list).

It is frequently the case in auction theory that results for two-bidder auctions may only extend to auctions where $n>2$ under more stringent conditions (if at all). This is such a situation, as the above conditions are unnecessary in two-bidder auctions:

Theorem 2 That $n=2$ is sufficient for $D B B$.

\footnotetext{
${ }^{4}$ For a random variable $W \sim H$, the survival function $\bar{H}(t)=\operatorname{Pr}\{W>t\} \forall t$.

5 Paul and Gutierrez (2004) obtain a corresponding characterization under a more stringent and less transparent assumption on the underlying stochastic order; their principal concern is with the relationship between the number of bidders and the expectation of the difference between the two highest order statistics.

${ }^{6}$ Log-concave density and IFR are related but not nested. If the density function $h$ is continuously differentiable and log-concave on $[a, b]$, then the distribution $H$ has IFR.

7 For completeness, for $U \sim H$, define $U_{(n+1)}=\inf \{t: U(t)>0\}$; this step assumes $X_{(n+1)}$ and $Z_{(n+1)}$ are finite, which is harmless for present purposes.

8 Sources: [a] Shaked and Shanthikumar 2006 Theorem 3.B.7; [b] Bartoszewicz 1986 Lemma 3(b); [c] Shaked and Shanthikumar 2006 Theorem 3.C.8; [d] Kochar et al. 2007 Theorem 3.

9 The weaker result is also in $\mathrm{Li}(2005)$.
} 
These three conditions suffice for dispersion to benefit bidders, but do not indicate the extent of the benefit. A fourth condition offers a measure of bidder benefit.

Theorem 3 That $X$ and $Y$ are normal is sufficient for DBB. Moreover, the increment of expected profit is proportional to the increment of the standard deviation.

None of these sufficient conditions are necessary. An abundance of cases exhibit DBB for arbitrary $n$, but no illuminating necessary condition seems possible.

It might appear that the expected difference between the two highest order statistics should always expand as variability increases. A counterexample: let $X \sim \log$-normal(2,4), $Y \sim N(15,5) ;$ a 10,000-iteration simulation with $n=50$ yields $E\left[X_{(1)}-X_{(2)}\right] \approx 8.49$, but $E\left[Z_{(1)}-Z_{(2)}\right] \approx 7.57$.

To understand how the example works, we introduce the concept of locally dispersive order. We say that $X$ is locally less dispersed than $Y$ on range subset $[\alpha, \beta]$ if $0<\alpha<\beta<1$ and $\exists d \in(-\alpha, 1-\beta)$ such that $F^{-1}(\beta)-F^{-1}(\alpha) \leq$ $G^{-1}(\beta+d)-G^{-1}(\alpha+d)$. Intuitively, the definition means that the C.D.F. of $Y$ is flatter than that of $X$ on some regions on their respective supports. In our problem, although $Z$ tends to be more dispersive than $X$ globally, the converse can happen locally. This leads to an algorithm to construct counterexamples: first, select $Y$ such that $Z$ is locally less dispersed than $X$; then select $n$ such that $X_{(1)}, X_{(2)}$, and $Z_{(1)}, Z_{(2)}$ fall in the corresponding regions. In the above counterexample, $E\left[X_{(1)}\right] \approx 18.90$ and $E\left[X_{(2)}\right] \approx 10.41$, both far in the tail of the distribution. Adding a normal distribution with a mean between $E\left[X_{(1)}\right]$ and $E\left[X_{(2)}\right]$ increases the variability, but also shifts rightward the log-normal distribution's head. The first- and second-order statistics are then in the resultant distribution's head. As the tail is much flatter than the head, the desired locally dispersive order is obtained. The general structure of cases reversing the DBB inequality eludes specification. The example demonstrates that dispersion does not always benefit the winning bidder.

\section{Expected Revenue}

As stated in the introduction, the bid-taker's expected revenue is the secondhighest expected value estimate. Continuing with $Z=X+Y, X, Y$ independent, we explore conditions yielding the characterization Dispersion Benefits Seller $(D B S)$, formally:

$$
E\left[X_{(2)}\right] \leq E\left[Z_{(2)}\right] .
$$

First, some intuition. Unlike $E\left[W_{(1)}-W_{(2)}\right]$, the expected value of the second-highest order statistic is clearly location dependent. For example, if $X \sim U[0,1]$ and $Y \sim U[-3,-2]$, then obviously $Z_{(2)} \leq-1$. Roughly speaking, the addition of $Y$ can be thought to have two effects. First, the mean of $Y$ : if $E[Y]>0$, it can "push up" $E\left[Z_{(2)}\right]$, making it larger. Inversely, $E[Y]<0$ 
may "drag it down." Second, the variability of $Y$ : it increases the variability of $Z$. This will approximately "push up" all order statistics above the mean and "push down" those below it. For $n>3$, greater variability tends to increase the second order statistic--usually above the mean.

Proposition 1 That $Y$ is nonnegative is sufficient for DBS.

It is trivial to construct a case in which DBS fails by choosing negative $Y$. To avoid trivialities, restrict attention to $Y$ with $E[Y] \geq 0$. When $n=2$, the second order statistic is below the mean as long as the distribution is not very right-skewed. To find a counter case is easy. When $n=3, E\left[Z_{(2)}\right]$ is the median. To construct a similar example, focus on the right-skewed distributions, as the mean exceeds the median. Let $X \sim \log$-normal $(10,10), T \sim \log$ normal $(10,100)$ and $Y=T-9$, so $E[Y]=1$. By 10,000-iteration simulation with $n=3, E\left[X_{(2)}\right] \approx 8.25<E\left[Z_{(2)}\right] \approx 3.69$. This inequality holds as well in simulations for $n=4,5,6$.

So examples of higher revenue with less dispersed information exist when $n \leq 6$. How about many bidders? Intuitively, the larger $n$ is, the more difficult to find such an example. While the natural conjecture is that examples may be constructed correspondingly, we know of no algorithm assuredly constructing examples for arbitrarily large $n .^{10}$

\section{Going Beyond Independent Randomnesses}

This section moves from independent randomness to the more complicated environments in which randomnesses are correlated. We consider a widely used case of the affiliation model: conditionally independent random variables. Let there be a random variable $V$, and let $\left(X_{1}, \ldots, X_{n}\right)$ and $\left(Y_{1}, \ldots, Y_{n}\right)$ be independent conditional on $V=v$ ( $Y$ being white noise is included as a special case).

The previous two sections utilized tools that link stochastic orders to order statistics under that assumption of independence; to our knowledge, there are not extant tools for analyzing affiliated variables. Accordingly, the characterizations here are slightly narrower than for independent types (although Proposition 1 extends by the same proof).

In general, if sufficient conditions in section 1 [2] above hold given $V=v \forall v$ for conditional variables $X$ and $Y$, then the same conditions are unconditionally sufficient for DBB [DBS] with affiliated values. These extensions follow from the relationship $E_{A}[A]=E_{B}[E[A \mid B]]$. To characterize fully general conditions under which sufficient conditions hold "for each $v$ " is elusive. Two broad, fairly general classes are treated in the following two theorems:

Theorem 4 If $X$ has an unconditional distribution that is hierarchical, that is, if $V$ is a parameter of the distribution of $X$, then sufficient conditions for

10 See appendix for a further discussion on several related questions. 
$D B B$ [DBS] under independent types remain sufficient when $X$ and $Y$ are independent conditional on $V$.

Letting $V$ be the asymptotic mean of $X$ is sufficient to satisfy Theorem 4 when $\left(X_{1}, \ldots, X_{n}\right)$ are exchangeable. For example, if $X \sim \operatorname{normal}(V, \sigma)$, then $X$ has $\log$-concave density for all $V=v$, extending DBB. Hierarchical distribution is sufficient but not necessary:

Theorem 5 If $X=W+g(V)$ for some nondecreasing function $g(\cdot)$, then either a log-concave density or IFR of $W_{i}$ 's is sufficient for $D B B$.

Proof $X+Y=W+g(V)+Y$. The claim follows immediately by treating $g(V)+Y$ as a random variable and applying Theorem 1.

A situation fitting Theorem 5 is when the private-value and common-value components are additive (as in, e.g., Pesendorfer and Swinkels 2000, or Goeree and Offerman 2003). ${ }^{11}$

Section 1 [2] provided examples in which dispersion harmed bidders [seller] when $X$ and $Y$ are independent. Corresponding examples for affiliated $X^{\prime}$ and $Y^{\prime}$ can easily be constructed in the following way: take any of the examples favoring the less dispersed environment for independent $X$ and $Y$. Letting $X^{\prime}=X$ and $Y^{\prime}=Y+\varepsilon X^{\prime}$ introduces affiliation. Letting $|\varepsilon|$ be arbitrarily small maintains affiliation and the inequality found above for that example.

\section{Concluding Remarks}

Under tenable assumptions for independent-types or affiliated-types environments, greater dispersion in bidders' information aids both bidders, via higher expected profit, and sellers, via higher expected revenue. Yet the needed assumptions are far from innocuous, as unusual but not pathological cases have been presented where greater variability in information can yield the reverse inequalities for expected differences between the two highest order statistics and for expectations of the second-highest value estimate.

There is reason to expect some robustness of the conclusions drawn, in such directions as small deviations from symmetric type distributions or from distributional families discussed. An example with sizable deviations from symmetric type distributions illustrates. Let types be drawn from uniform distributions: $X_{1} \sim U[2,6], X_{2} \sim U[3,6], X_{3} \sim U[4,6], X_{4} \sim U[5,6]$ and the mean-preserving spreads $Z_{1} \sim U[1,7], Z_{2} \sim U[2,7], Z_{3} \sim U[3,7], Z_{4} \sim$ $U[4,7]$. The more dispersed environment exhibits a greater expected profit, $E\left[X_{(1)}-X_{(2)}\right]=0.45<E\left[Z_{(1)}-Z_{(2)}\right]=0.83$, and a greater expected revenue, $E\left[X_{(2)}\right]=5.21<E\left[Z_{(2)}\right]=5.34$. The greater expected profit in the higher variability environment wouldn't necessarily be an expectation each bidder could face, but in this example, conditional on winning, each bidder's expected profit is higher with type distributions $\left(Z_{j}\right)$ than with $\left(X_{j}\right) .{ }^{12}$

\footnotetext{
11 The appendix considers situations where affiliated types do not satisfy conditional independence.

12 A related example is in the appendix.
} 
As noted, often bid-takers may have auction design decisions and informationdisclosure policy decisions that can impact type variability. There may also be scope for decisions by bidders that have information-dispersion aspects. Matthews (1977, 1984) and Bergemann and Valimaki (2002) consider models in which bidders decide how precise an estimate of asset value to acquire, at a cost; more precise estimates are likely to yield the environments considered here to have less dispersed types. In their models, ex-ante symmetric information-improving technologies lead to equilibria in which information precision is symmetric. The results above suggest the circumstances in which greater information-improving efforts might coincide with lesser (or greater) dispersion of resulting asset value estimates and thus with lesser or greater gradients of expected profitability. Further, they suggest when a seller might have an incentive to subsidize (or, if feasible, to tax) bidders' information-improving costs.

\section{Appendix}

Proof of Theorem 2: Construct trial observations of $\mathfrak{Z}$ as follows. Take 2 draws of $X$, denoted $x_{(1)}, x_{(2)}$ (descendingly), and 2 draws of $Y$, denoted by $y_{(1)}, y_{(2)}$. Next, randomly assign $y_{(i)}$ 's to $x_{(i)}$ 's, $i=1,2$. It is equally likely that $x_{(1)}$ is associated with $y_{(1)}$ or $y_{(2)}$, each having probability 0.5 . First, suppose $x_{(1)}$ is associated with $y_{(1)}$, so $x_{(2)}$ is associated with $y_{(2)}$. Recall $z_{(1)}=x_{(1)}+y_{(1)}$ and $z_{(2)}=x_{(2)}+y_{(2)}$, so $\Delta z=z_{(1)}-z_{(2)}=\left(x_{(1)}-\right.$ $\left.x_{(2)}\right)+\left(y_{(1)}-y_{(2)}\right)=\Delta x+\Delta y$. Second, if instead $x_{(1)}$ is associated with $y_{(2)}$, then $x_{(2)}$ is associated with $y_{(1)}$. It is then unclear which of $x_{(1)}+y_{(2)}$ and $x_{(2)}+y_{(1)}$ is bigger, but clear that $\Delta z=\left|\left(x_{(1)}+y_{(2)}\right)-\left(x_{(2)}+y_{(1)}\right)\right|=|\Delta x-\Delta y|$. Hence the expected value of $\Delta z=0.5(\Delta x+\Delta y)+0.5|\Delta x-\Delta y| \geq 0.5(\Delta x+\Delta y)+0.5(\Delta x-\Delta y)=\Delta x$, namely $\Delta z \geq \Delta x$ in each such constructed trial. Then, by the law of large numbers, $E[\Delta Z]=$ $\lim _{n \rightarrow \infty} \frac{\sum_{k=1}^{n} E\left[\Delta z_{k}\right]}{n} \geq \lim _{n \rightarrow \infty} \frac{\sum_{k=1}^{n} \Delta x_{k}}{n}=E[\Delta X]$.

Proof of Theorem 3: Letting $Z=\stackrel{n}{\alpha} X+\beta$, where $\alpha$ is a positive constant, it is straightforward that

1. $Z_{(i)} \triangleq \alpha X_{(i)}+\beta$ for all $i$, and ${ }^{13}$

2. $Z_{(1)}-Z_{(2)} \triangleq \alpha\left[X_{(1)}-X_{(2)}\right]$.

If $\alpha=1$ and $\beta=-\mu=-E[X]$, then $Z_{(1)}-Z_{(2)}=X_{(1)}-X_{(2)}$ for $Z=X-\mu$. Thus the difference between the first- and second-order statistics is location independent. Normalizing the means of the random variables to 0 is without loss of generality.

To show Theorem 3, assume both $X$ and $Y$ have means 0. By closure of normal distributions under addition, $Z=X+Y$ is also normally distributed with mean 0 . Let $\alpha=\sqrt{\sigma_{x}^{2}+\sigma_{y}^{2}} / \sigma_{x}$, then $\alpha X$ is a normal random variable having the same mean and variance as $Z$. Hence, $Z \triangleq \alpha X$, so $E\left[Z_{(1)}-Z_{(2)}\right]=\alpha E\left[X_{(1)}-X_{(2)}\right]$.

Proof of Proposition 1: Let $x_{1}, \ldots, x_{n}$ be a size- $n$ realization of $X$, and $x_{(1)}, x_{(2)}$ the two largest values. Adding a size- $n$ realization of $Y$ to $x_{1}, \ldots, x_{n}$ yields $z_{1}, \ldots, z_{n}$. Suppose $x_{(1)}, x_{(2)}$ become $x_{(1)}+\xi, x_{(2)}+\tau$, where $\xi, \tau \geq 0$ by assumption. Clearly, $\left\{z_{(1)}, z_{(2)}\right\}$ are not necessarily $\left\{x_{(1)}+\xi, x_{(2)}+\tau\right\}$. If $z_{(i)}=x_{(2)}+\tau$ for some $i=2, \ldots, n$, then $z_{(2)} \geq z_{(i)}=$ $x_{(2)}+\tau \geq x_{(2)}$. If $z_{(1)}=x_{(2)}+\tau$, then $z_{(2)} \geq x_{(1)}+\xi \geq x_{(2)}$. Since this is true in each realization, $E\left[X_{(2)}\right] \leq E\left[Z_{(2)}\right]$. Note that the proof does not require $Y$ to be independent of $X$.

Further notes on Section 2: An example: If $X \sim N\left(\mu_{x}, \sigma_{x}^{2}\right), Y \sim N\left(\mu_{y}, \sigma_{y}^{2}\right)$, a sufficient condition for DBS is that $\left(1-\sqrt{\sigma_{x}^{2}+\sigma_{y}^{2}} / \sigma_{x}\right) \mu_{x}+\mu_{y}>0$. To see this, note that by closure

13 The sign "ㅇ" means "equal in distribution". 
under addition, $Z=X+Y$ is also a normal. Let $\alpha=\sqrt{\sigma_{x}^{2}+\sigma_{y}^{2}} / \sigma_{x}$ and $\beta=(1-\alpha) \mu_{x}+\mu_{y}$, then $Z \triangleq \alpha X+\beta$. Note that $\alpha \geq 1$. If $\beta>0$, then $E\left[Z_{(2)}\right]=\alpha E\left[X_{(2)}\right]+\beta>E\left[X_{(2)}\right]$.

Several questions: (1) Does DBS follow if $X$ is symmetric, $Y$ has mean 0 , and $n>3$ ? No, an example: $X \sim[1+N(0,1)]$ and $Y \sim \log -\operatorname{normal}(2,4)-2, E\left[Z_{(2)}\right] \approx 0.98<$ $E\left[X_{(2)}\right] \approx 1.30$. This is due to the skewness of $Y$. (2) Does DBS follow if in addition $Y$ is symmetric? While our conjectured answer is no, no verifying example is at hand. However, if $X$ has a log-concave density, then DBS follows, since the space between any two adjacent order statistics increases (this is preliminary [b] in the proof of Theorem 1 ).

Further notes on Section 3: That the joint distribution $(X, Y)$ is bivariate normal is sufficient for DBB. If, in addition, $\left(1-\sqrt{\sigma_{x}^{2}+\sigma_{y}^{2}+2 \rho_{x y} \sigma_{x} \sigma_{y}} / \sigma_{x}\right) \mu_{x}+\mu_{y}>0$, (where $\mu_{x}, \mu_{y}, \sigma_{x}, \sigma_{y}, \rho_{x y}$ are the means, standard deviations, and correlation coefficient), DBS follows. To see this, note that if the joint distribution $(X, Y)$ is bivariate normal, then $Z=X+Y$ is normal. Letting $\alpha=\sqrt{\sigma_{x}^{2}+\sigma_{y}^{2}+2 \rho_{x y} \sigma_{x} \sigma_{y}} / \sigma_{x}$ and $\beta=(1-\alpha) \mu_{x}+\mu_{y}$ yields $Z \triangleq \alpha X+\beta$. DBB follows since $\alpha \geq 1$. In addition, if $\beta>0$, then $E\left[Z_{(2)}\right]>E\left[X_{(2)}\right]$.

An alternative construction is specialized, but not distribution-specific. Let $X$ and $Y$ be independent, and assume that for $Z=X+Y$, sufficient conditions for DBB and DBS apply (from any results in sections 1 and 2). Construct $X^{\prime}=X$ and

$$
Y^{\prime}=\left\{\begin{array}{l}
\alpha X^{\prime}+\beta, \text { with probability } p \\
Y, \text { with probability } 1-p
\end{array},\right.
$$

where $\alpha, \beta \geq 0, p \in(0,1)$. By construction, $X^{\prime}$ and $Y^{\prime}$ are affiliated. Then,

$$
Z^{\prime}=X^{\prime}+Y^{\prime}=\left\{\begin{array}{l}
(\alpha+1) X+\beta, \text { with probability } p \\
X+Y, \text { with probability } 1-p
\end{array} .\right.
$$

and $\alpha^{\prime}=\alpha+1 \geq 1, \beta \geq 0$ suffice for DBB and DBS in this affiliation setting.

Another example of asymmetric distributions: Types are draws from normal distributions: $X_{1} \sim N(10,4.5), X_{2} \sim N(10.5,4), X_{3} \sim N(11,3.5), X_{4} \sim N(11.5,3)$, and the variability that the less dispersed environment adds is symmetric: $Z_{j}=X_{j}+Y$, with $Y \sim N(0,5)$, again a mean-preserving spread. This example reaches the same comparisons: $E\left[X_{(1)}-X_{(2)}\right]=2.71<E\left[Z_{(1)}-Z_{(2)}\right]=4.53$, and $E\left[X_{(2)}\right]=11.93<E\left[Z_{(2)}\right]=$ 12.67 .

\section{References}

1. Bagnoli, Mark and Ted Bergstrom, 2005, Log-concave probability and its applications, Economic Theory, 26, pp. 445-469.

2. Bartoszewicz, Jaroslaw, 1986, Dispersive ordering and the total time on test transformation, Statistics $\&$ Probability Letters, 4, pp. 285-288.

3. Bergemann, Dirk, and Juuso Valimaki, 2002, Information acquisition and efficient auction design, Econometrica, 70(3), pp. 1007-1033.

4. Goeree, Jacob, and Theo Offerman, 2003, Competitive bidding in auctions with private and common values, Economic Journal, 113, pp. 1-20.

5. Kochar, Subhash, Xiaohu Li, and Maochao Xu, 2007, Excess wealth order and sample spacings, Statistical Methodology, 4, pp. 385-392.

6. Li, Xiaohu, 2005, A note on expected rent in auction theory, Operations Research Letters 33 , pp. $531-534$.

7. Mares, Vlad, and Ronald M. Harstad, 2007, Ex-post full surplus extraction, straightforwardly, Economic Theory, 32, pp. 399-410.

8. Matthews, Steven, 1977, Information acquisition in competitive bidding processes, working paper, California Institute of Technology.

9. Matthews, Steven, 1984, Information acquisition in discriminatory auctions, Bayesian Models in Economic Theory, M. Boyer and R. E. Kihlstrom, eds. North-Holland, pp. 181-207. 
10. Myerson, Roger B., 1981, Optimal auction design, Math of Oper. Res., 6, pp. 58-73.

11. Paul, Anand, and Genaro Gutierrez, 2004, Mean sample spacings, sample size and variability in an auction-theoretic framework, Operations Research Letters, 32, pp. 103-108.

12. Pesendorfer, Wolfgang, and Jeroen Swinkels, 2000, Efficiency and information aggregation in auctions, American Economic Review, 90, pp. 499-525.

13. Ross, S. M., 1996, Stochastic Processes (2nd ed.), Wiley, New York.

14. Shaked, M., 1982, Dispersive Ordering of Distributions, Journal of Applied Probability, Vol. 19, No. 2, pp. 310-320

15. Shaked, M. and J. G. Shanthikumar, 2006, Stochastic Orders, Springer Verlag, New York. 